\title{
Field-current phase diagrams of in-plane spin transfer torque memory cells with low effective magnetization storage layers
}

Cite as: J. Appl. Phys. 115, 17C713 (2014); https://doi.org/10.1063/1.4862842

Submitted: 23 September 2013 . Accepted: 24 October 2013 . Published Online: 28 January 2014

L. San Emeterio Alvarez, B. Lacoste, B. Rodmacq, L. E. Nistor, M. Pakala, R. C. Sousa, and B. Dieny
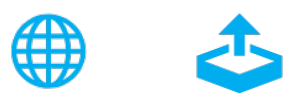

View Online

Export Citation

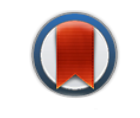

CrossMark

\section{ARTICLES YOU MAY BE INTERESTED IN}

Perpendicular spin transfer torque magnetic random access memories with high spin torque efficiency and thermal stability for embedded applications (invited)

Journal of Applied Physics 115, 172615 (2014); https://doi.org/10.1063/1.4870917

$\mathrm{X}$-ray magnetic circular dichroism for $\mathrm{Co}_{x} \mathrm{Fe}_{4-x} \mathrm{~N}(\mathrm{x}=0,3,4)$ films grown by molecular beam epitaxy

Journal of Applied Physics 115, 17C712 (2014); https://doi.org/10.1063/1.4862517

Size dependence of spin-torque induced magnetic switching in CoFeB-based perpendicular magnetization tunnel junctions (invited)

Journal of Applied Physics 111, $07 C 711$ (2012); https://doi.org/10.1063/1.3677385

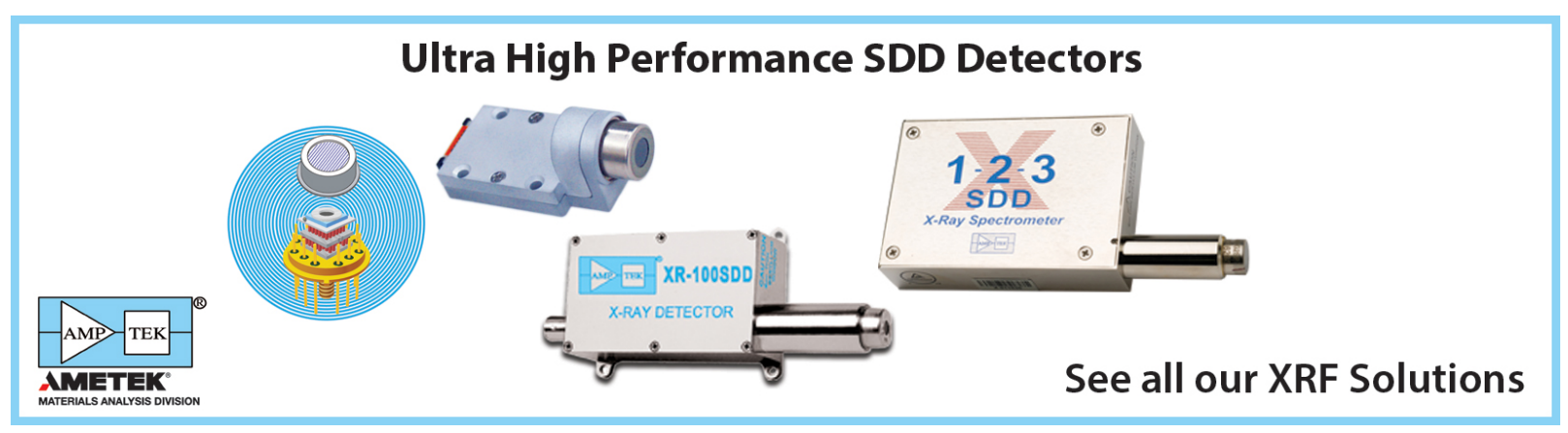




\title{
Field-current phase diagrams of in-plane spin transfer torque memory cells with low effective magnetization storage layers
}

\author{
L. San Emeterio Alvarez, ${ }^{1}$ B. Lacoste,${ }^{1}$ B. Rodmacq,${ }^{1}$ L. E. Nistor, ${ }^{2}$ M. Pakala, ${ }^{3}$ \\ R. C. Sousa, ${ }^{1, a)}$ and B. Dieny ${ }^{1}$ \\ ${ }^{1}$ SPINTEC, UMR(8191) CEA/CNRS/UJF/Grenoble INP; INAC, 17 rue des Martyrs, 38054 Grenoble, France \\ ${ }^{2}$ Applied Materials SGG CTO, Les Jardins d'Entreprise 11b, Chemin de la Dhuy 38246 Meylan Cedex, France \\ ${ }^{3}$ Applied Materials SSG CTO, 974 E Arques avenue, Sunnyvale, California 94085, USA
}

(Presented 6 November 2013; received 23 September 2013; accepted 24 October 2013; published online 28 January 2014)

\begin{abstract}
Field-current phase diagrams were measured on in-plane anisotropy $\mathrm{Co}_{60} \mathrm{Fe}_{20} \mathrm{~B}_{20}$ magnetic tunnel junctions to obtain the spin transfer torque (STT) field-current switching window. These measurements were used to characterise junctions with varying free layer thicknesses from 2.5 down to $1.1 \mathrm{~nm}$ having a reduced effective demagnetizing field due to the perpendicular magnetic anisotropy at $\mathrm{CoFeB} / \mathrm{MgO}$ interface. Diagrams were obtained with $100 \mathrm{~ns}$ current pulses, of either same or alternating polarity. When consecutive pulses have the same polarity, it is possible to realize the STT switching even for conditions having a low switching probability. This was evidenced in diagrams with consecutive pulses of alternating polarity, with $100 \%$ switching obtained at $4.7 \mathrm{MA} / \mathrm{cm}^{2}$, compared to the lower $3.4 \mathrm{MA} / \mathrm{cm}^{2}$ value for same polarity pulses. Although the low level of the current density window is higher in alternating polarity diagrams, the field window in both diagrams is the same and therefore independent of the pulse polarity sequence. (C) 2014 AIP Publishing LLC. [http://dx.doi.org/10.1063/1.4862842]
\end{abstract}

Recent work on magnetic random access memories (MRAM) has demonstrated the possibility of reducing the switching critical current for in-plane anisotropy cells, through the reduction of the effective field acting on the storage layer by creating a perpendicular magnetic anisotropy in the free layer stack. This was first demonstrated in spin-valve systems ${ }^{1}$ and then in magnetic tunnel junctions. ${ }^{2}$ The critical current density for reversal through spin transfer torque, $j_{s w}$, is mainly determined by the demagnetizing field $H_{d}$. The general target current density is below $1 \mathrm{MA} / \mathrm{cm}^{2}$, such that a cell with an area of $100 \times 100 \mathrm{~nm}^{2}$ can be switched with a current lower than $100 \mu \mathrm{A}$. The selection transistor connected to the MRAM cell could then have the same approximate lateral dimension as the magnetic dot size. The interest of the low demagnetization approach lies in the possibility to significantly reduce the critical current without impacting the cell thermal stability, assuming that all other material parameters remain unchanged. The equation for the critical switching current $j_{s w}$ in the absence of thermal fluctuations can be written as ${ }^{3}$

$$
j_{s w}=\frac{2 e \alpha \mu_{0} M_{S} t_{F}}{\hbar \eta}\left(H_{a p p}+H_{\text {coup }}+H_{k}+\frac{1}{2} H_{e f f}\right),
$$

where $\alpha$ is the free layer damping, $t_{F}$ is the free layer thickness and $M_{s}$ the saturation magnetization, such that the critical current depends linearly on $M_{s} t$. The shape anisotropy in the case of a flat elliptical cell can be written as ${ }^{4}$ $H_{k}=4 \pi M_{s}\left(\frac{t}{w}\right)\left(n_{y}-n_{x}\right)$, with $n_{x, y}$ the demagnetizing factors along the easy and hard axis, respectively. Finally, $H_{\text {eff }}$ is the effective demagnetizing field equal to $H_{d}-H_{k \perp}$, where the perpendicular demagnetizing field is $H_{d} \approx 4 \pi M_{s}$ and $H_{k \perp}$

${ }^{\text {a)} E l e c t r o n i c ~ m a i l: ~ r i c a r d o . s o u s a @ c e a . f r . ~}$ is the induced perpendicular anisotropy field. In MTJs, this perpendicular anisotropy can have different origins: either bulk as in Rare Earth transition metal alloys or interfacial such as at $\mathrm{Co} / \mathrm{Ni}, \mathrm{Co} / \mathrm{Pt}$ or $\mathrm{Co}(\mathrm{Fe})(\mathrm{B}) /$ oxide interfaces. ${ }^{5}$ In the case of $\mathrm{CoFeB} / \mathrm{MgO}$ based magnetic tunnel junctions, the reduction of the demagnetizing field becomes significant as the thickness of the free layer is reduced. In this paper, we have investigated spin transfer torque (STT) switching of $\mathrm{Co}_{60} \mathrm{Fe}_{20} \mathrm{~B}_{20}$ which presents a reduced perpendicular demagnetizing field, obtained by reducing the thickness of the free layer from 2.5 to $1.1 \mathrm{~nm}$. The critical switching current was obtained from a field-current phase diagram, where it is possible to highlight the precise dependence of STT switching as a function of the in-plane applied field and current density.

Magnetic tunnel junctions were deposited by an Endura PVD sputtering tool of Applied Materials on $300 \mathrm{~mm} \mathrm{Si} / \mathrm{SiO}_{2}$ wafers. The stack of the in-plane MTJ structures is: a pinned reference layer of $\mathrm{PtMn} / \mathrm{Co}_{70} \mathrm{Fe}_{30} / \mathrm{Ru} / \mathrm{Co}_{60} \mathrm{Fe}_{20} \mathrm{~B}_{20}(2.5 \mathrm{~nm})$, $1.2 \mathrm{~nm} \mathrm{MgO}$ barrier deposited by RF sputtering and a free storage layer $\mathrm{Co}_{60} \mathrm{Fe}_{20} \mathrm{~B}_{20}$ having different thicknesses from 1 up to $2.5 \mathrm{~nm}$. The TMR and RA product measured at different sites on the wafer and on patterned pillars are shown in Figure 1. There is a decrease in RA from center to edge, suggesting a thinner $\mathrm{MgO}$ thickness at the edge, corresponding to a thicknesses difference of $0.1 \mathrm{~nm}$. Sample pieces of $4 \times 4 \mathrm{~cm}^{2}$ were cut from four wafers with different $\mathrm{CoFeB}$ thickness of 1.1 , $1.4,1.8$, and $2.5 \mathrm{~nm}$ for nano-patterning. These sample cuts were also measured by CIPT. The films were patterned to elliptical dots from $50 \times 100 \mathrm{~nm}^{2}$ up to $100 \times 200 \mathrm{~nm}^{2}$ using e-beam lithography followed by reactive ion etching and ion beam milling. After patterning the measured RA was in the 10 to $13 \Omega \mu \mathrm{m}^{2}$ range. This compares to $\sim 15 \Omega \mu \mathrm{m}^{2}$ of the MRCIP measurement, which was used for all further 

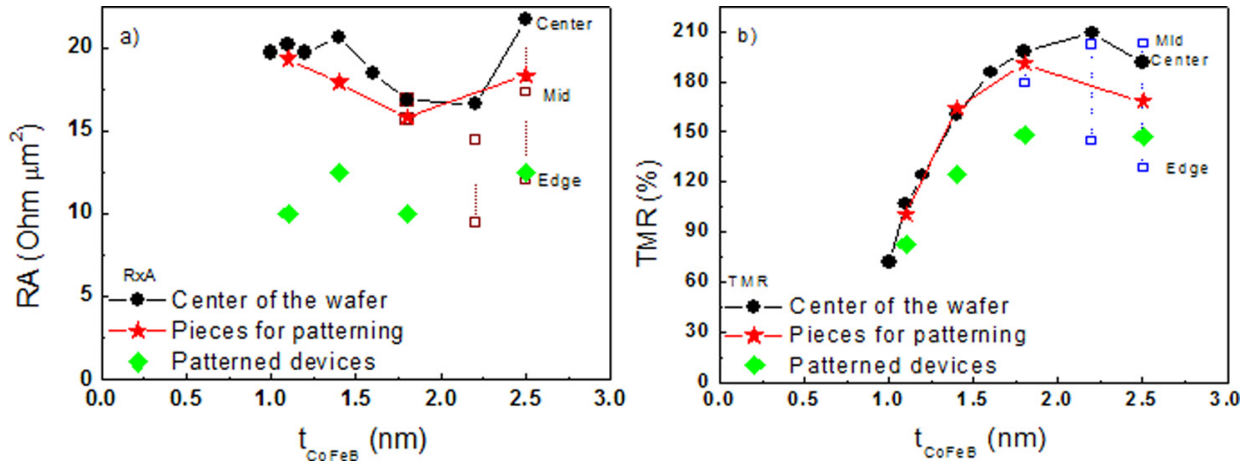

FIG. 1. (a) RA values for the center of the $300 \mathrm{~mm}$ wafers (black circles), the $4 \times 4 \mathrm{~cm}^{2}$ pieces cut from the middle of the wafer (red stars), and the patterned devices (green squares) for different $\mathrm{CoFeB}$ thicknesses. (b) Values of TMR for both center $(0 \mathrm{~cm})$, middle $(6 \mathrm{~cm})$, and edge $(12 \mathrm{~cm})$ of the wafer versus thickness. The results on patterned dots show smaller RA and TMR values due to difference in nominal and actual dot size in the determination of the RA product. calculations of current density, so that differences between nominal and actual dot size do not influence current density calculations. The stacks with $2.5 \mathrm{~nm}$ CoFeB show $160 \%$ TMR on continuous films $\left(4 \times 4 \mathrm{~cm}^{2}\right.$ slices) and a maximum $144 \%$ after patterning. Reducing the $\mathrm{CoFeB}$ free layer thickness results in lower TMR, which results from a reduction of the spin polarization $(\mathrm{P})$, translating also into a lower spin-transfer efficiency parameter $(\eta)$ in eq1. The TMR measured by MRCIP at $1.1 \mathrm{~nm}$ $\mathrm{CoFeB}$ is still $100 \%$ on wafers and $83 \%$ on patterned junctions.

Vibrating sample magnetometer measurements show a reduction of the free layer effective demagnetizing field $\left(H_{\text {eff }}\right)$ as the CoFeB thickness is decreased from 2.5 down to $1.1 \mathrm{~nm}$. This reduction is ascribed to the perpendicular $\mathrm{MgO} / \mathrm{CoFeB}$ interfacial anisotropy which partially counterbalances the easy-plane demagnetizing energy. This field decreases from $18 \mathrm{kOe}$ for $2.5 \mathrm{~nm}$ (the demagnetizing field value for $\mathrm{Co}_{60} \mathrm{Fe}_{20} \mathrm{~B}$ ) down to $3.6 \mathrm{kOe}$ for the thinnest $1.1 \mathrm{~nm}$ free layer, as shown in Figure 2. (H,I) state phase diagrams were obtained on patterned junctions, measuring the tunnel junction resistance at each field step after applying a $100 \mathrm{~ns}$ current pulse for increasing pulse amplitudes. A full hysteresis loop is recorded at a given pulse amplitude for both current polarities. The resistance measurement is performed at $30 \mathrm{mV}$ bias to prevent heating in the junction. In our measurement convention, positive voltage favours the antiparallel (AP) state while negative voltage favours the parallel (P) state. A complete phase diagram recorded for pulse amplitudes from $0.1 \mathrm{~V}$ up to $1 \mathrm{~V}$ in $100 \mathrm{mV}$ steps is shown in Figure 3(a), which

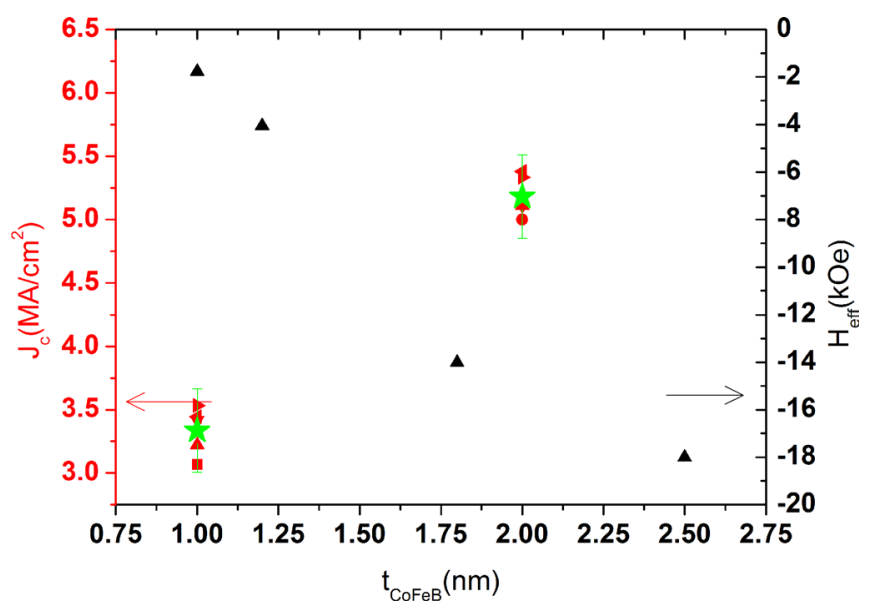

FIG. 2. Dependence of the effective demagnetizing field (black triangles) and critical current density on the free layer thickness. The average value of different size junctions is plotted as green stars, while the red points are an average of different junctions having the same size. corresponds to a 50 by $300 \mathrm{~nm}^{2}$ junction with $1.1 \mathrm{~nm} \mathrm{CoFeB}$ free layer. The colour code represents the resistance of the junction at a given field point, using the average of the increasing and decreasing field sweeps. Low and high resistances are coded as blue (P-state) and red (AP-state). The green area corresponds to the average between minimum and maximum values, representing the hysteresis loop bi-stable region. The effect of STT is clearly visible in the bi-stable region, as the AP state is favoured for positive pulse voltage and P state for negative polarity. The results show also a reduction of the switching field from $\mathrm{P}$ to AP (AP to P) under positive (negative) voltage, showing an approximately linear dependence with the applied voltage, corresponding to a perpendicular spin torque or field-like term. ${ }^{6}$ Its pulse bias dependence is represented by the dashed lines on the diagram, corresponding to a slope of approximately $-50 \mathrm{Oe} / \mathrm{V}$ for both polarities. These values compare to reported slopes of $-88 \mathrm{Oe} / \mathrm{V}$, observed on free layers of $\mathrm{Co}_{49} \mathrm{Fe}_{21} \mathrm{~B}_{30} 2 \mathrm{~nm}^{7}$ In our presented data only the $\mathrm{P}$ or AP states are favoured depending on the pulse polarity, however, some junctions would also present back-hopping or switch-back effect, where the reversal from the STT favoured state is observed as reported in other studies. ${ }^{8}$

To obtain the critical switching voltage, the absolute resistance change observed between positive and negative polarity was plotted in the switching diagram of Fig. 3(b). This region defines the working field-voltage window for STT switching. The critical current density was calculated from the minimum voltage point where the full resistance change can be observed, $0.5 \mathrm{~V}$ in the shown diagram. The breakdown voltage observed is above $1.3 \mathrm{~V}$ for same polarity consecutive pulses. The data values for different free layer thicknesses are shown in Figure 2. where a reduction of the critical current density from $5.8 \mathrm{MA} / \mathrm{cm}^{2}$ for a free layer of $1.8 \mathrm{~nm}$ to 3.4 $\mathrm{MA} / \mathrm{cm}^{2}$ at $1.1 \mathrm{~nm}$ is observed. This is lower than the ratio of the measured perpendicular saturation fields and free layer thicknesses. At a reduced thickness, two factors contribute to the increase of the switching current density. The first is a reduction of the spin torque efficiency due to lower spin polarization, as observed in the lower TMR values. The second, more significant, is a higher damping value, which could increase by a factor of 3 , as reported by Ikeda. ${ }^{9}$

The field-voltage diagrams shown in Figure 3 can be used to identify the STT working window, but contain no information on the switching probability at a given applied voltage. It is also possible to apply consecutive pulses of alternating polarity as the field is swept and repeat this measurement for a number of write attempts to obtain the switching probability as 

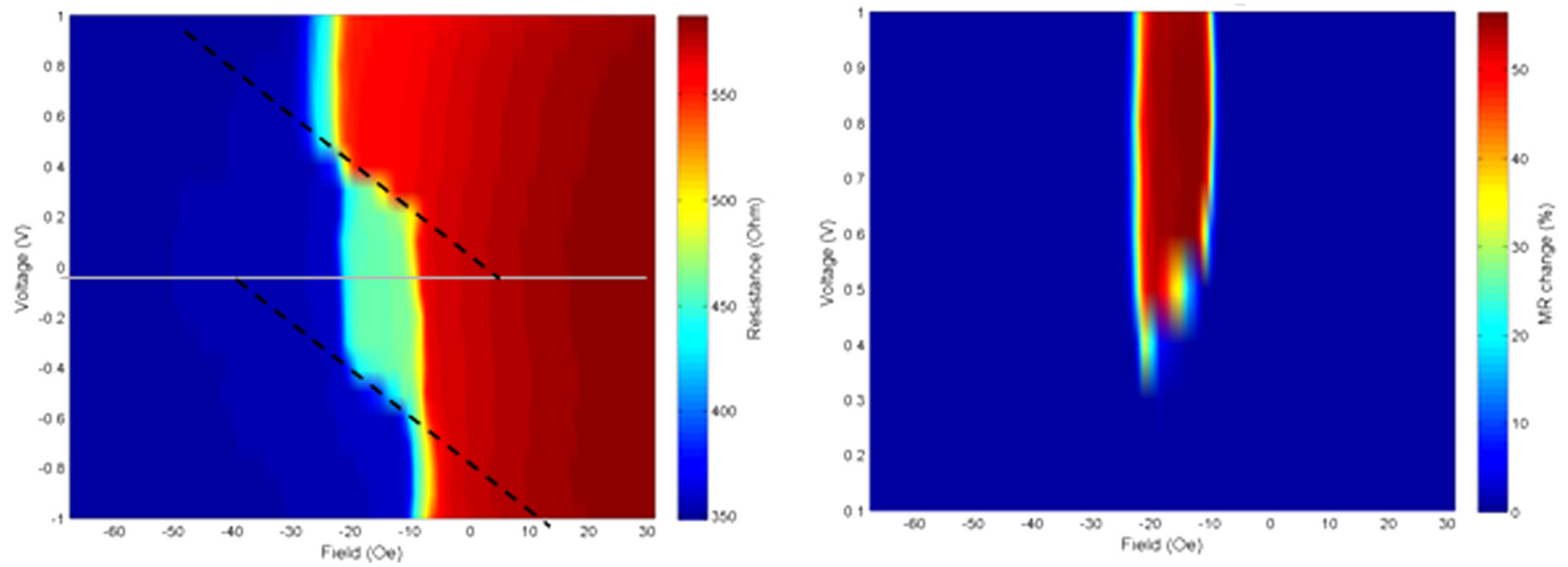

FIG. 3. Field and current phase diagrams corresponding to the average of 50 loops measured while applying $100 \mathrm{~ns}$ pulses. a) Blue (left area of the diagram) corresponds to the low resistance parallel P-state, red (right area of the diagram) corresponds to the high resistance antiparallel AP-state and the green (middle area) corresponds to the bi-stable region. b) Diagram representing the difference (calculated as \% change) between resistance values at positive and negative voltage at the same field value. The red area (middle) corresponds to the current-field window where STT switching is possible from P to AP state is possible. A similar window exists at negative voltage for AP to P.

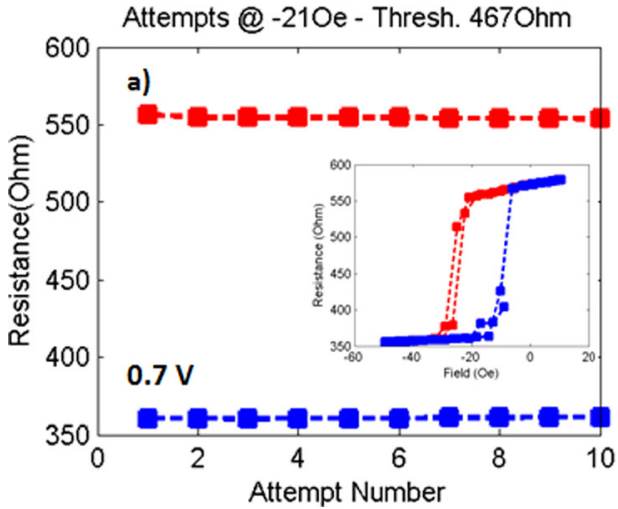

function of the field and voltage. Figure 4 shows 10 write attempts for an applied bias field of -21 Oe. It becomes then clear that the switching probability of pulses with the minimum writing voltage of $0.5 \mathrm{~V}$ is only $\sim 30 \%$ and successful switching in all 10 attempts is only obtained at $0.7 \mathrm{~V}$. When consecutive pulses of the same polarity are continuously applied, switching does occur even at low switching probability voltage levels. By applying a sequence of same polarity pulses irreversible switching can occur even at low probability current levels, resulting in current levels similar to those obtained in current sweep measurements. The insets in Figure 4 show the hysteresis loop for positive or negative applied pulses, each point being the average of the 10 writing attempts. At $0.7 \mathrm{~V}$, there is a match between the STT field range of Figure 3(b) and the field region encompassed by the positive and negative voltage hysteresis loops.

In conclusion, we have shown that field-current switching phase diagrams applying pulses of different voltage amplitudes clearly show the field-current window where STT switching can be realised. These measurements were used to characterise $\mathrm{Co}_{60} \mathrm{Fe}_{20} \mathrm{~B}_{20}$ based tunnel junctions with in-plane anisotropy and reduced effective field as thickness is decreased to $1.1 \mathrm{~nm}$. For diagrams where the same pulse polarity is applied at every field value, the critical current value obtained corresponds to a low switching probability condition. This is evidenced in diagrams with consecutive pulses of alternating polarity. At alternating polarity $100 \%$ switching is only obtained at $4.7 \mathrm{MA} / \mathrm{cm}^{2}$, while the same polarity diagrams show a lower $3.4 \mathrm{MA} / \mathrm{cm}^{2}$ value. The low level of the current density window is higher using alternating polarity diagrams compared to the same polarity. However, in both types of diagrams, the field window is exactly the same and therefore independent of the pulse polarity.

This work was partially supported by the PATHOS project of the French National Research Agency ANR. We would like to acknowledge L. Vila for e-beam lithography.

${ }^{1}$ L. Liu et al., Appl. Phys. Lett. 94, 122508 (2009).

${ }^{2}$ P. Khalili Amiri et al., Appl. Phys. Lett. 98, 112507 (2011).

${ }^{3}$ S. Mangin et al., Nature Mater 5, 210 (2006).

${ }^{4}$ J. Z. Sun et al., Appl. Phys. Lett. 78, 4004 (2001).

${ }^{5}$ S. Monso et al., Appl. Phys. Lett. 80, 4157 (2002).

${ }^{6}$ S.-C. Oh et al., Nat. Phys. 5, 898 (2009).

${ }^{7}$ S.-Y. Park et al., Phys. Rev. B 84, 214417 (2011).

${ }^{8}$ T. Min et al., J. Appl. Phys. 105, 07D126 (2009).

${ }^{9}$ Ikeda et al., Nature Mater 9, 721 (2010). 